\title{
A review on rheological properties and measurements of dough and gluten
}

\begin{abstract}
The field of rheology has seen a wider application in the food industry recently although, it is a complex concept and that most food systems possess non-ideal characteristics. Nevertheless, the rheological behavior of foods are able to be determined using various techniques and equipment. Studies on rheological properties related to dough and gluten are often challenging due to its variance in nature and high dependence on many factors. This study attempts to give a review on the various types of experimental techniques and set-up used in quantifying rheological properties of dough and gluten. The rheological properties are defined and the behaviors are described by inducing stress and strains in small and large deformation studies.
\end{abstract}

Keyword: Deformation; Dough; Food rheology; Gluten; Rheological measurement 